\title{
Extension of the KDO turbulence/transition model to account for roughness
}

\author{
Chunfei Fang ${ }^{1}$ and Jinglei $X u^{2,3^{*}}$
}

\section{${ }^{*}$ Correspondence:}

xujl@buaa.edu.cn

${ }^{2}$ School of Energy and Power

Engineering, Beihang

University, Beijing 100191,

China

Full list of author information

is available at the end of the

article

\begin{abstract}
Wall roughness significantly influences both laminar-turbulent transition process and fully developed turbulence. A wall roughness extension for the KDO turbulence/ transition model is developed. The roughness effect is introduced via the modification of the $k$ and $v_{t}$ boundary conditions. The wall is considered to be lifted to a higher position. The difference between the original position and the higher position, named as equivalent roughness height, is linked to the actual roughness height. The ratio between the two heights is determined by reasoning. With such a roughness extension, the predictions of the KDO RANS model agree well with the measurements of turbulent boundary layer with a sand grain surface, while the KDO transition model yields accurate cross-flow transition predictions of flow past a 6:1 spheroid.
\end{abstract}

Keywords: Transition model, Turbulence model, Cross-flow transition, Wall roughness

\section{Introduction}

Computational Fluid Dynamics (CFD) is widely used as a predictive tool for fluid motions. Fluids in nature and engineering applications often interact with solid walls. Neither natural walls nor man-made walls are perfectly smooth. Wall roughness significantly affects laminar-turbulence transition process. After transition, turbulence on a rough surface is enhanced compared with that on a smooth one, leading to higher skin frictions and heat transfer rates. Therefore, it is necessary to account for roughness in CFD simulations.

It is unrealistic to set up a graphical CAD model with every roughness-element for a CFD simulation. Thus, it is assumed that the roughness-element size in any direction is small compared with the boundary layer thickness so that, above the roughnesses, the flow is averaged over numerous roughness elements that exact location of which is not accounted for [1]. The wall presented in the computational domain is smooth, and the velocity on the wall is also zero. To account for the effect of roughness, the "equivalent sand grain" approach [2] is commonly employed. This approach links the real surface to a sand grain surface by converting the real roughness height to the equivalent sand grain height with the help of empirical correlations [3, 4]. The correlations consider a variety of real roughness shapes. The correlations proposed by Dirling [3] and Grabow and White [4] established the general paradigm of wall roughness modeling. The basic idea is author(s) and the source, provide a link to the Creative Commons licence, and indicate if changes were made. The images or other third party material in this article are included in the article's Creative Commons licence, unless indicated otherwise in a credit line to the material. If material is not included in the article's Creative Commons licence and your intended use is not permitted by statutory regulation or exceeds the permitted use, you will need to obtain permission directly from the copyright holder. To view a copy of this licence, visit http:// creativecommons.org/licenses/by/4.0/. 
to mimic roughness effect by increasing the turbulent eddy viscosity or turbulent energy in the wall region. The increment is determined by the equivalent sand grain height.

This equivalent sand grain approach is successful in predicting fully developed turbulence. However, to capture the laminar-turbulent transition process which is influenced by roughness effect, additional modeling techniques must be supplemented. Xiang et al. [5] proposed a hypersonic cross-flow transition criterion considering surface roughness, yet no roughness modeling for fully developed turbulence was presented. Liu et al. [6] employed the Wilcox wall boundary condition for $\omega$ [7] to introduce roughness effect for fully developed turbulence. Liu et al. [6] also employed an additional transport equation of $A_{r}$, the roughness amplification factor, to reflect roughness effect in the laminarturbulent transition process. $A_{r}$ mainly affects the $\gamma$ equation, and thus $A_{r}$ is a roughness correction for the transition equation.

According to the previous work, it is evident that two roughness corrections are needed: one on the turbulent equations $(k, \omega)$, and one on the transition equations $(\gamma)$. The roughness correction on the turbulent equations is a relatively mature one which, in fact, leads to enhanced turbulent viscosity. The enhanced turbulent viscosity itself is capable of reflecting the premature transition process. Thus, it is possible for turbulence models with wall roughness corrections to be capable of capturing the influence of roughness on the transition process, without additional roughness corrections to transition equations. This work aims to develop such a model, the existence of which supports the viewpoint that laminar, transitional, and turbulent flows are ONE.

\section{Transition/turbulence model with roughness consideration}

\subsection{Baseline model}

The Kinetic energy Dependent Only model (KDO) [8] adopts a physics-based formulation, with less empiricism, for Reynolds Averaged Navier-Stokes Equation closure. For transition predictions, KDO does not explicitly model a specific transition mechanism but can capture many types of transition phenomena. Along with the evolution of the turbulent kinetic energy $(k)$ equation, transition phenomena naturally appear, which is similar to what Navier-Stokes equations do. The key is that all the model elements for the RANS closure are flow-structure-adaptive. The model equations read,

$$
\begin{aligned}
& k_{, t}+\left(U_{j} k\right)_{, j}=-\overline{u_{i}^{\prime} u_{j}^{\prime}} U_{i, j}+\left[\left(\nu+v_{t}\right) k_{, j}\right]_{, j}-\varepsilon \\
& \varepsilon=\varepsilon_{1}+\varepsilon_{2} \\
& \varepsilon_{1}=2 v \sqrt{k_{, j}} \sqrt{k}, j
\end{aligned}
$$

The turbulence Reynolds number, $R e_{k}$ can be used for model calibrations. $R e_{k}$ is defined as $R e_{k}=\sqrt{k} d / v$, in which $d$ is the wall distance. It is obvious that $R e_{k}$ is a local variable and such a variable is beneficial to parallel computation.

$$
\begin{aligned}
& \text { For } R e_{k}<10, \\
& \qquad \varepsilon_{2}=A_{\varepsilon} k^{3 / 2} / d
\end{aligned}
$$




$$
A_{\varepsilon}=1.34\left(\operatorname{Re}_{k} / 0.25\right)^{-0.8}\left(1+\left(\operatorname{Re}_{k} / 0.25\right)^{1.5}\right)^{0.45 / 1.5}\left(1+\left(\operatorname{Re}_{k} / 2.4\right)^{1.5}\right)^{-0.1 / 1.5}
$$

For $R e_{k} \geq 10$,

$$
\begin{aligned}
& \varepsilon_{2}=\min \left(A_{\varepsilon}, 0.8\right) k^{3 / 2} \max \left(1 / L,\left(1-\mathrm{e}^{-R e_{k} / 1300}\right) / d\right) \\
& A_{\varepsilon}=1.4\left(\operatorname{Re}_{k} / 4.3\right)^{-1.9}\left(1+\left(\operatorname{Re}_{k} / 4.3\right)^{5}\right)^{0.2 / 5}\left(1+\left(R e_{k} / 28\right)^{4.9}\right)^{2.76 / 4.9} \\
& \left(1+\left(\operatorname{Re}_{k} / 66\right)^{10}\right)^{-0.15 / 10}\left(1+\left(\operatorname{Re}_{k} / 110\right)^{10}\right)^{0.12 / 10}\left(1+\left(\operatorname{Re}_{k} / 175\right)^{10}\right)^{-0.4 / 10} \\
& L=\sqrt{\Omega_{i} \cdot \Omega_{i}} / \sqrt{\nabla_{j} \Omega_{i} \cdot \nabla_{j} \Omega_{i}}, \quad \Omega_{i}=\nabla \times \mathbf{U}
\end{aligned}
$$

By extending Bradshaw's assumption down to the wall, the Reynolds stress constitutive relation is established as,

$$
\begin{aligned}
& R_{b}=\tau_{12} / k \\
& -\overline{u_{i}^{\prime} u_{j}^{\prime}}=R_{b} k \frac{2 S_{i j}}{S}, \quad S=\sqrt{2 S_{i j} S_{i j}}
\end{aligned}
$$

where $\tau_{12}$ is the principal Reynolds shear stress, $-\overline{u_{i}^{\prime} u_{j}^{\prime}}$ is the Reynolds stress tensor, $k$ is the turbulent kinetic energy, $R_{b}$ is the Bradshaw's coefficient, and $S_{i j}$ is the mean strain rate tensor.

For the KDO turbulence model [8], the Bradshaw's coefficient reads,

$$
R_{b}=\min \left[0.018\left(\operatorname{Re}_{k} / 1\right)^{0.56}\left(1+\left(\operatorname{Re}_{k} / 120\right)^{2.5}\right)^{-0.56 / 2.5}\left(1+\left(\operatorname{Re}_{k} / 225\right)^{10}\right)^{0.05 / 10}, 0.283\right]
$$

in which, $R e_{k}=\rho \sqrt{k} d / \mu$ is the turbulent Reynolds number. For the KDO transition model [9], the Bradshaw's coefficient reads,

$$
R_{b}=\min \left[0.1165(r / 1)^{0.37}\left(1+(r / 1)^{1.15}\right)^{-0.157 / 1.15}\left(1+(r / 72)^{2}\right)^{-0.213 / 2}, 0.283\right]
$$

in which, $r=\mu_{\mathrm{t}} / \mu$ is eddy viscosity ratio. The eddy viscosity ratio, a measurement of the intensity of turbulence, is a transport variable. Due to the transport properties of $r, R_{b}$ is capable of capturing transition phenomena, such as bypass transition, natural transition, separation induced transition and cross flow transition. To conclude, the KDO model is one turbulence model that could predict both fully turbulent flows and laminar-turbulent flow transitions, by solving only the $k$ equation. The information of turbulence and laminar-turbulent flow transition is included in the $k$ equation, and there is no such distinction as turbulent equation or intermittency equation. Therefore, a typical roughness correction imposed on the KDO model could potentially reflect the roughness effects on the transition process. 


\subsection{Roughness extension}

The idea of "equivalent sand grain approach" is employed here. The basic idea is that, according to experimental data [2], the log-law still holds in a turbulent boundary layer with wall roughness. The difference is that, the $y^{+}-U^{+}$profile moves upwards, leading to a shifted log-law,

$$
u^{+}=\frac{1}{\kappa} \ln \frac{y^{+}}{h_{s}^{+}}+C
$$

where $h_{s}$ is the equivalent sand grain height. In Eq. (13), $y$ and $h_{s}$ are non-dimensioned by $v / u_{\tau}$. For example, $y^{+}$equals $y u_{\tau} / v$, in which $u_{\tau}=\sqrt{\tau_{w} / \rho}$ is the wall friction velocity. The position of the wall can be considered to be raised from $y$ to $y+d_{0}$. $d_{0}$, named as the equivalent roughness height, is the roughness height for CFD computations. $d_{0}$ is less than $h_{s}$ due to that there are spaces among sand elements. On the other hand, roughness enhances the turbulent viscosity to a value that is much larger than the molecular viscosity on the wall, and we have,

$$
u_{\tau}^{2}=v_{t} \frac{\partial U}{\partial y}=u_{\tau} \kappa\left(y+d_{0}\right) \frac{\partial U}{\partial y}
$$

Note that Eq. (14) assumes that the boundary layer is fully turbulent, so Eq. (14) might not be valid for transitional flows. The solution of Eq. (14) is,

$$
u^{+}=\frac{1}{\kappa}\left[\ln \left(y+d_{0}\right)-\ln \left(d_{0}\right)\right]
$$

By substituting Eq. (13) to Eq. (15), it is easy to obtain the relationship between $d_{0}$ and $h_{s}$.

$$
d_{0}=\exp (-C \kappa) h_{s}
$$

Aupoix and Spalart [1] set $C$ to 8.5 , which is valid for very rough surfaces. In such a roughness model, $d_{0}$ equals $0.03 h_{s}$, and this is inconsistent with the intuition that $d_{0}$ and $h_{s}$ should be of the same magnitude. On the other hand, Chedevergne and Aupoix [10] stated that $C$ can range from 5.5 to 9.7, indicating that even if the log-law still holds, the universality is compromised.

The present work employs the idea of "equivalent sand grain approach", but the empirical coefficient is determined by reasoning instead of the shifted log-law. This work also employs the equivalent roughness height $d_{0}$. The relation between $d_{0}$ and $h_{s}$ is also

$$
d_{0}=C_{r} h_{s}
$$

If the real roughness height $h$ is known,

$$
d_{0}=C_{r}^{0} h
$$

The original wall distance, $d$, in the KDO turbulence model is replayed by,

$$
\tilde{d}=d+d_{0}
$$


For a surface uniformly covered with sand grain, considering that the sand grain is with spherical shape, $C_{r}$ is set to 0.35 . For a surface that experienced polishing treatment, the rough elements are uniformly distributed on the surface, so $C_{r}^{0}$ is around 0.5 . The two constants are assessed in the following sections via CFD simulations.

The roughness effect is introduced via boundary conditions. The turbulent kinetic energy, $k$, on a smooth wall is zero. But for a rough wall, with the definition $k^{+}=k / u_{\tau}^{2}$,

$$
k_{w}=\left.k^{+}\right|_{w} u_{\tau}^{2}=\left.\left.k^{+}\right|_{w}\left(v+v_{t}\right) \frac{\partial U}{\partial y}\right|_{w}
$$

$k^{+}$can be expressed by $y^{+}$, and a model is calibrated by flat plate boundary layer at $R e_{\theta}=4060[11]$,

$$
\begin{gathered}
k^{+}=f\left(y^{+}\right)=0.131\left(y^{+} / 1\right)^{2}\left(1+\left(y^{+} / 3\right)^{1.6}\right)^{-0.5 / 1.6}\left(1+\left(y^{+} / 8\right)^{3.9}\right)^{-1.38 / 3.9} \\
\left(1+\left(y^{+} / 19\right)^{5.4}\right)^{-0.46 / 5.4}\left(1+\left(y^{+} / 40\right)^{4}\right)^{0.18 / 4}
\end{gathered}
$$

As seen in Fig. 1, Eq. (21) is valid in the range $0<y^{+}(1)<10^{3}$, which covers the viscous layer, the buffer layer, and the log layer. Along with the increment of $y^{+}$, the flow undergoes laminar state, laminar-turbulent transition, and turbulent state, indicating that Eq. (21) could potentially capture all the flow states. For a rough wall, $\left.k^{+}\right|_{w}$ in Eq. (20) is calculated by,

$$
\begin{array}{r}
\left.k^{+}\right|_{w}=0.131\left(d_{0}^{+} / 1\right)^{2}\left(1+\left(d_{0}^{+} / 3\right)^{1.6}\right)^{-0.5 / 1.6}\left(1+\left(d_{0}^{+} / 8\right)^{3.9}\right)^{-1.38 / 3.9} \\
\left(1+\left(d_{0}^{+} / 19\right)^{5.4}\right)^{-0.46 / 5.4}\left(1+\left(d_{0}^{+} / 40\right)^{4}\right)^{0.18 / 4}
\end{array}
$$

On a smooth wall $\left.v_{t}\right|_{w}$ is set to 0 . As to $\left.v_{t}\right|_{w}$ in Eq. (20), a Neumann boundary condition is employed,

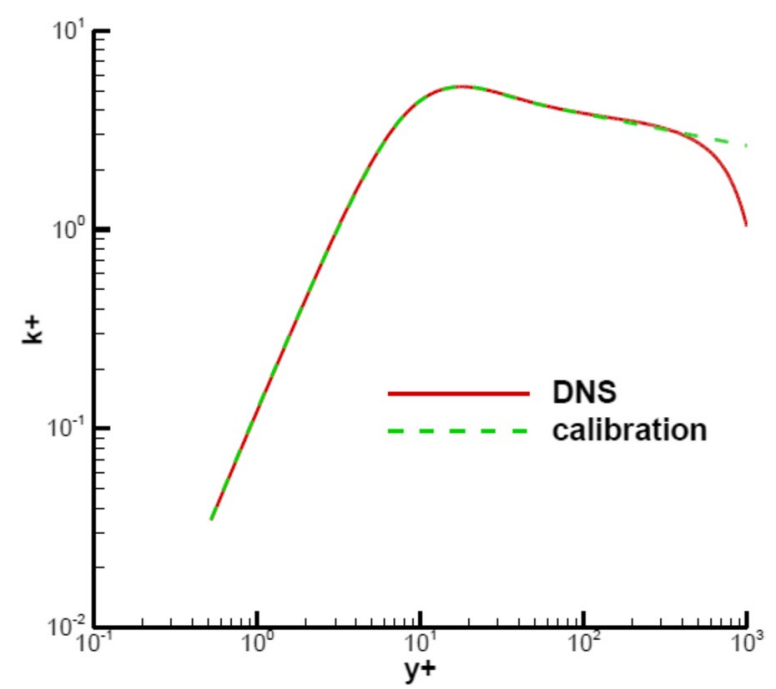

Fig. $1 y^{+}-k^{+}$profile calibration 


$$
\left.\frac{\partial v_{t}}{\partial n}\right|_{w}=\frac{v_{t}}{\tilde{d}}
$$

The KDO RANS model [8] with this roughness correction is termed as KDOR. The KDO transition model [9] (KDO-tran) with this roughness correction is termed as KDOR-tran.

\section{Computational results}

\subsection{Turbulent boundary layer}

Blanchard [12] conducted experiments over various surfaces. The turbulence on a sand grain paper, the average height of which is $0.425 \mathrm{~mm}$, is often studied as a benchmark test case. The case corresponds to a zero pressure gradient flow, with an external velocity of $45 \mathrm{~m} / \mathrm{s}$. Since the experiment focused on the roughness effects on fully developed turbulence, the KDOR model is employed. Figure 2 shows the skin friction distributions on the wall. The results of KDOR agree well with the experimental data, with $C_{r}=0.35$, which corresponds to sand grain surface. The classic result of the roughness-extended SA model [1], termed as Boeing, is also shown as a reference. The KDO model yields much lower skin friction, which corresponds to a turbulent boundary layer on a smooth wall. Different values of $C_{r}$ can yield different skin friction distributions. A sensitivity study of $C_{r}$ is shown in Fig. 3. It is obvious that along with the increment of $C_{r}$, the predicted skin friction gradually increases. The KDOR model yields the best accurate predictions, indicating that the optimal value of $C_{r}$ is 0.35 .

To confirm the optimal value of $C_{r}$, the experimental data of Hosni et al. [13] is employed as another benchmark test case. In the turbulence modeling study of Suga et al. [14], the freestream velocity is $58 \mathrm{~m} / \mathrm{s}$ and the equivalent sand grain height is $0.63 \mathrm{~mm}$. This work adopts the same settings. The predictions of KDOR (with $C_{r}=0.35$ ) again agree well with the experimental data, shown in Fig. 4. The experiment even provided a velocity profile at $x=0.86 \mathrm{~m}$, which is shown in Fig. 5 . It is clear that the surface

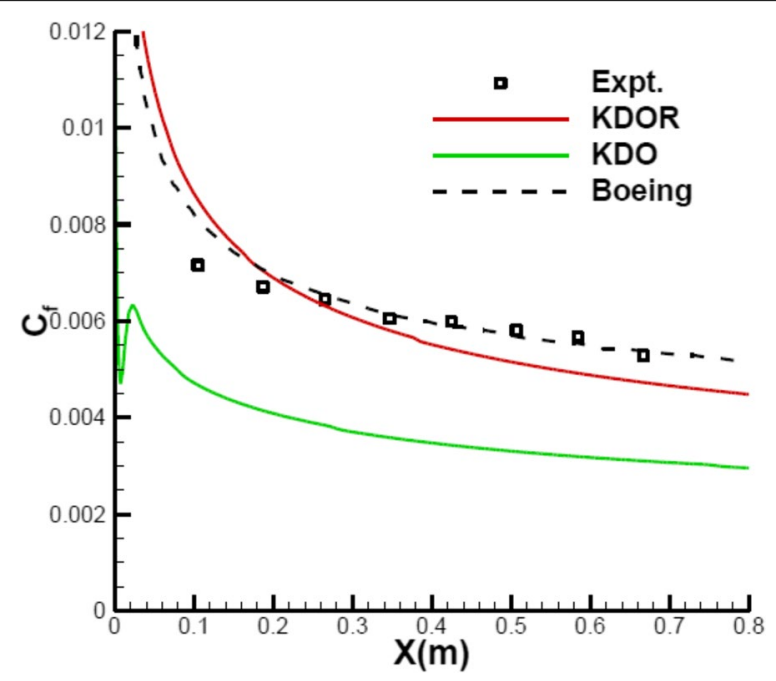

Fig. 2 Skin friction on the surface 


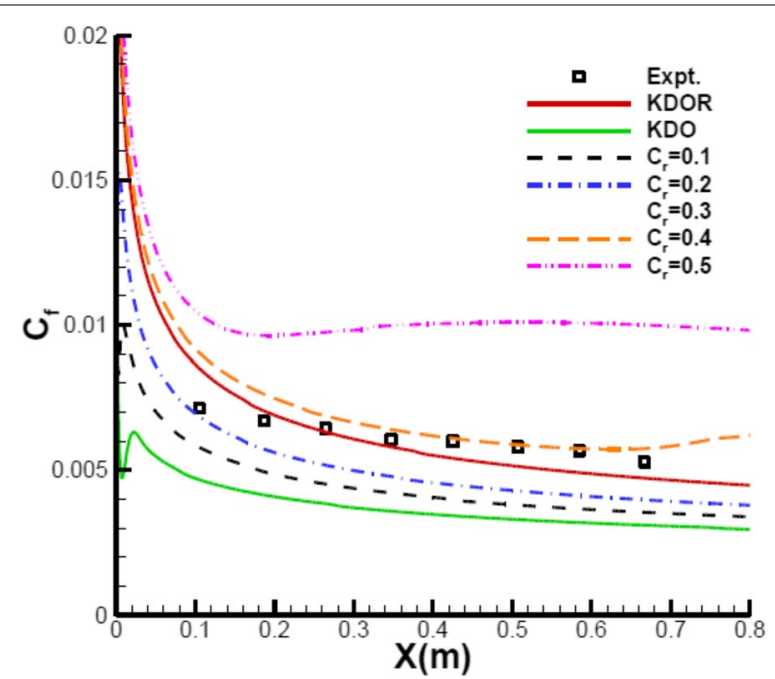

Fig. 3 Effect of $C_{r}$ on skin friction

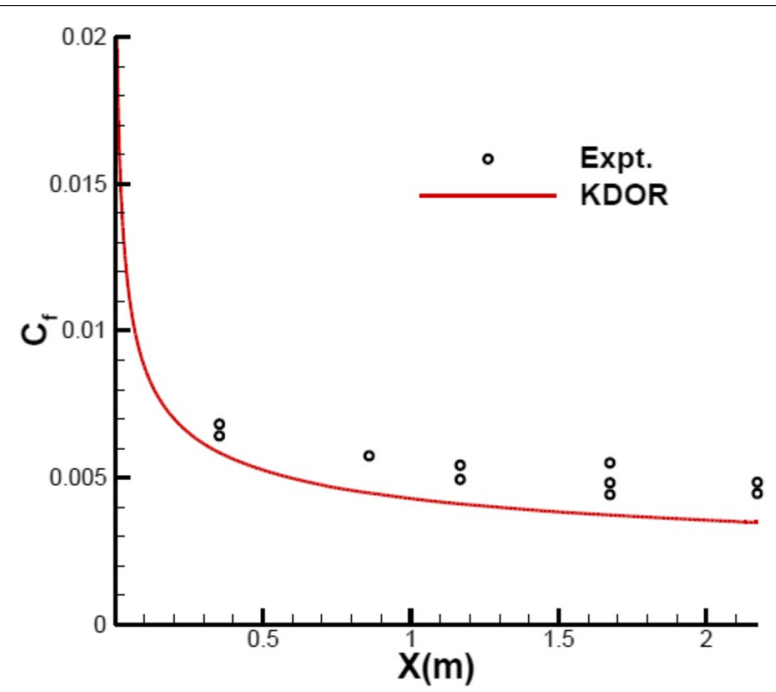

Fig. 4 Skin friction on the surface

roughness yields a shifted log-law velocity profile. The KDOR model, the predictions of which agree with the measurements very well, has captured this feature.

\subsection{Cross-flow transition on a spheroid}

DVLR (now DLR) conducted experiments [15] on flow past a 6:1 spheroid at various Reynolds numbers and attack angles. The long diameter was $2.4 \mathrm{~m}$ and the short diameter was $0.6 \mathrm{~m}$. The real roughness height, $h$, was about $3.3 \mu \mathrm{m}$. The attack angle is set to $15^{\circ}$ and the Reynolds number based on the long diameter is set to $6.5 \times 10^{6}$. The flow was nearly incompressible and the Mach number is set to 0.2 . This test condition was extensively studied by various transition models. The inflow turbulence intensity, $\mathrm{Tu}$, is a controversial one. In the literature, Tu can range from $0.1 \%$ to $1 \%$. A discussion with 


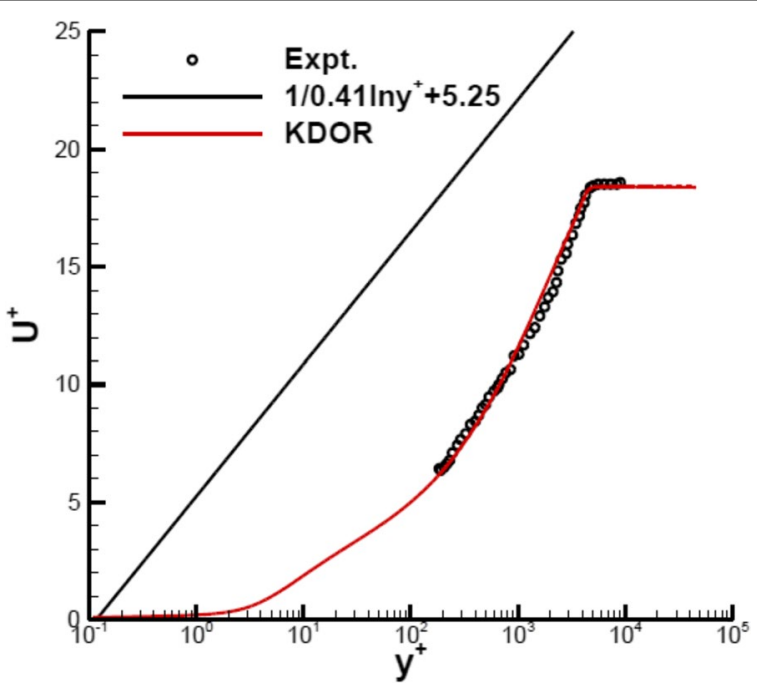

Fig. 5 Stream-wise velocity profile

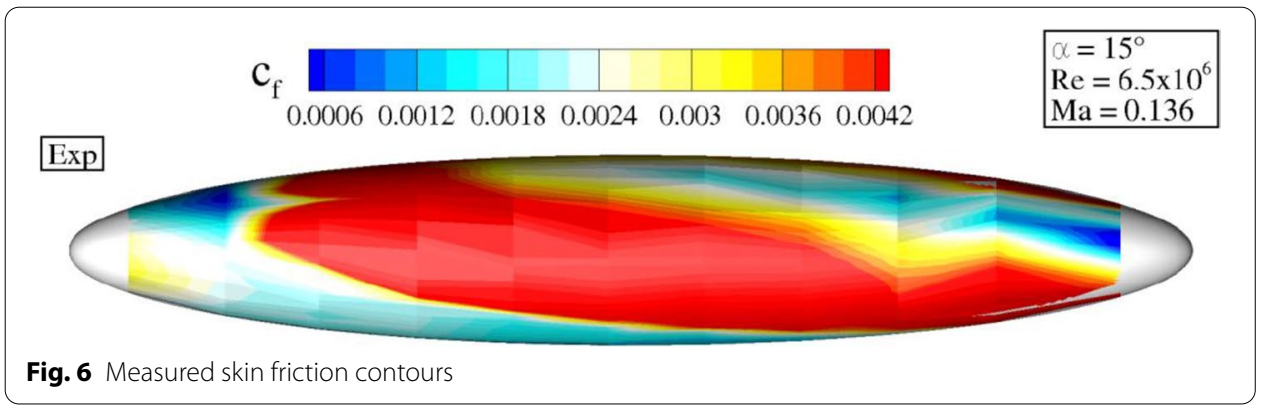

the researchers from DLR confirmed us that Tu was about $0.2 \%$, which is employed here. Since the surface of the spheroid was polished rather than covered with sand grain and the real roughness height is known, it is preferred that $C_{r}^{0}$ be 0.50 . However, to explore the influence of $C_{r}^{0}$ on the transition pattern, values 0.35 and 0.00 are tested. $C_{r}^{0}=0.00$ corresponds to a spheroid with a perfectly smooth surface. Since it is a cross-flow transition case, the KDOR-tran model is employed. The cross-flow transition pattern on the spheroid is illustrated by the skin friction contours. The experimental result is shown in Fig. 6. Figures 7, 8, and 9 show the results of KDOR-tran with $C_{r}^{0}$ being equal to 0.50 , 0.35 , and 0.00 , respectively. It is clear that the cross-flow transition pattern agrees with the measurements very well when $C_{r}^{0}$ equals 0.50 . However, the transition onset location is slightly delayed compared with the measurements. A slightly increased Tu could optimize the predictions, but this work insists the value of $0.2 \%$ provided by DLR. When $C_{r}^{0}$ reduces to 0.35 , the cross-flow transition pattern begins to deviate from the true pattern. When $C_{r}^{0}$ reduces to 0.00 , the transition onset locations are greatly delayed and the transition pattern differs a lot from the true pattern. It is necessary to point out that the $C_{r}^{0}=0.00$ and $\mathrm{Tu}=0.2 \%$ condition yields laminar flows on the surface, and the results in Fig. 9 correspond to the $C_{r}^{0}=0.00$ and $\mathrm{Tu}=0.6 \%$ condition. The skin friction distributions on the $z=0$ and $y=0$ slices of the spheroid are shown in Figs. 10 


$$
1
$$




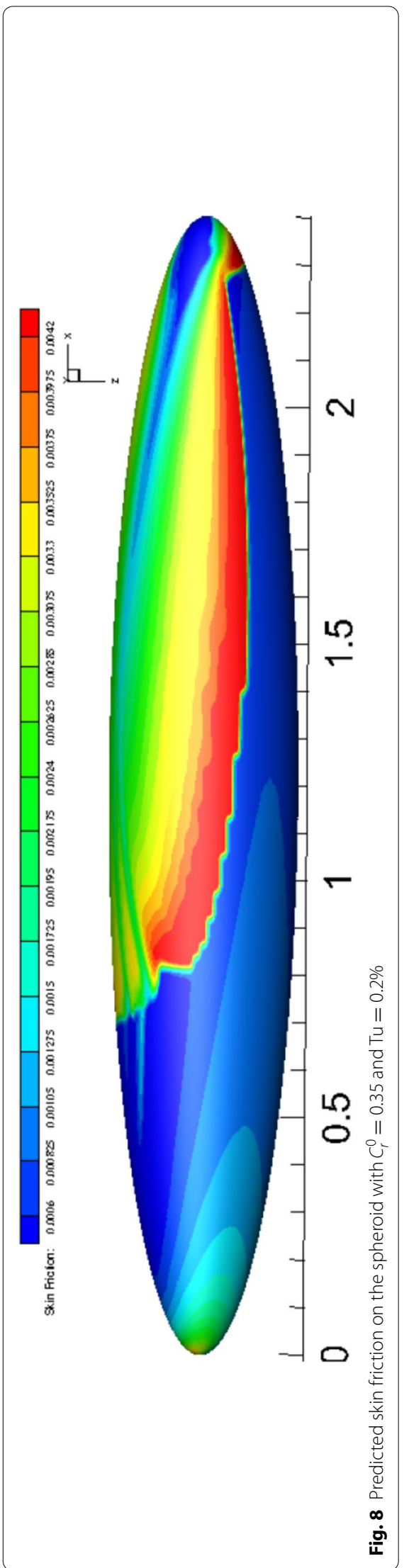




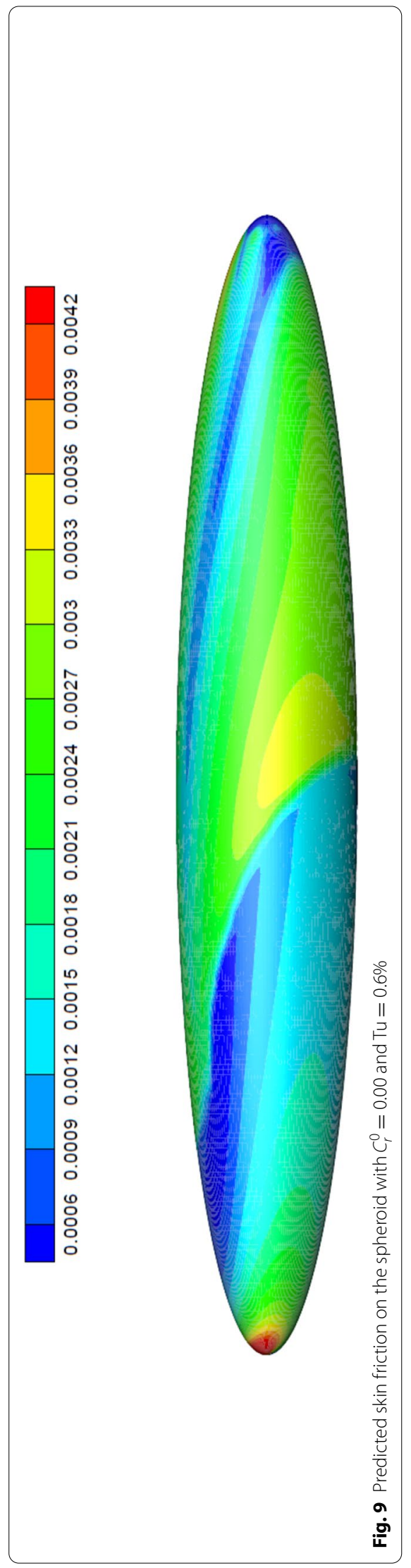




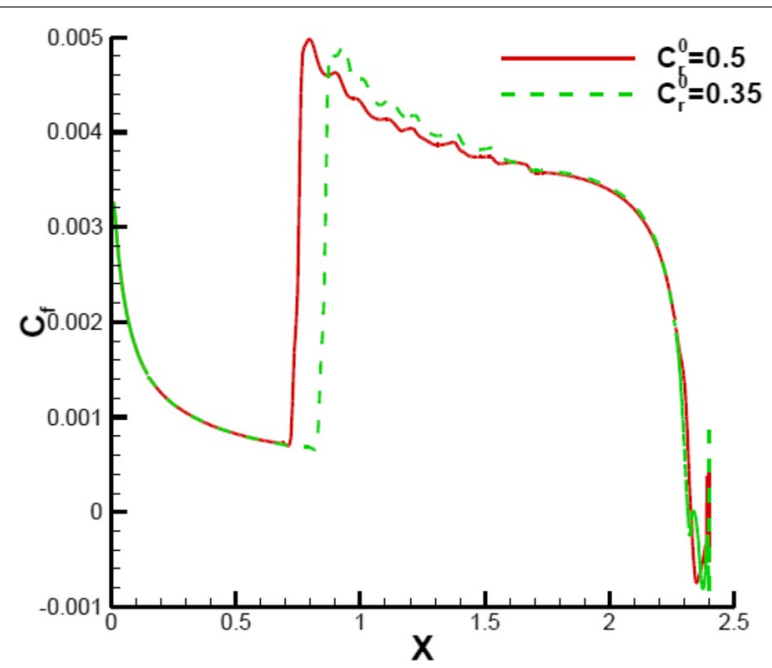

Fig. 10 Skin friction on $z=0$ slice

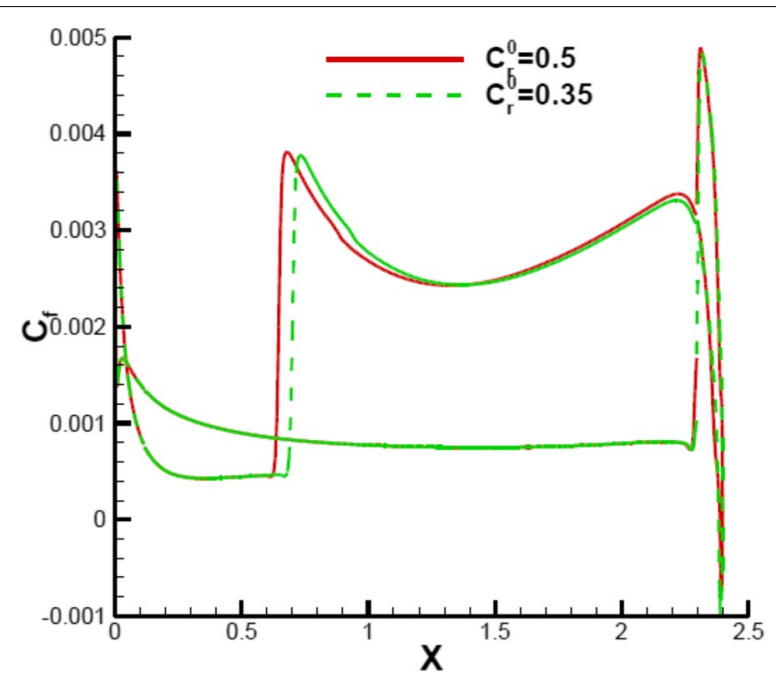

Fig. 11 Skin friction on $y=0$ slice

and 11, respectively. The $z=0$ slice corresponds to the centerline while the $y=0$ slice corresponds to the top and bottom lines on the spheroid. It is clear $C_{r}^{0}=0.35$ leads to delayed transition onset positions compared with the predictions of $C_{r}^{0}=0.5$. For the $z=0$ slice, the distributions of skin friction become wavy after transition, indicating that strong unsteadiness appears. For both slices, the skin frictions become negative at the end of the spheroid, indicating there are separations. According to the simulations, wall roughness does play an important role in the laminar-turbulence transition process, and it is necessary to take into account the roughness effects in turbulence modeling. 


\section{Conclusions}

A roughness extension of the KDO turbulence/transition model has been derived. It assumes non-zero viscosity and turbulent kinetic energy at the wall and it changes the definition of the wall distance, $d$. Thus, CFD code developers need only to alter the boundary conditions. The governing equations remain unchanged. Unlike the classic roughness extensions which utilize the altered log-law to calibrate empirical coefficients, this extension uses reasoning as the empiricism. The ratio between the equivalent roughness height and the sand grain roughness height is 0.35 . The ratio between the equivalent roughness height and the real roughness height is 0.5 . The ratios indicate that these roughness heights are of the same order, so the ratios are reasonable. In addition, the ratios are both verified by the CFD simulations.

Test on a flat plate boundary layer with sand grain surface shows that the KDOR model can well predict fully developed turbulence. Test on a spheroid with polished surface shows that the KDOR-tran model is capable of capturing cross-flow transition with wall roughness. The two models both employ the new roughness extension. The key formula of the roughness extension is a $y^{+}-k^{+}$distribution, which is obtained from the DNS data of a smooth flat plate. Surprisingly, the formula works well for rough walls, indicating the formula is of universality. The roughness elements in the test cases are small enough to be hidden in the region $y^{+}<300$. The region is dominated by the wall, and the $y^{+}-$ $k^{+}$distribution is insensitive to stream-wise pressure gradient. Therefore, the roughness extension of the KDO model can be used for various complex boundary layer flows with rough surfaces. For larger roughness elements, the roughness extended KDO model is well worth a try.

To conclude, this work has successfully developed a roughness extension for the KDO turbulence/transition model. With such an extension, the KDO model is capable of capturing not only fully developed turbulence, but also the influence of roughness on the transition process, without additional roughness corrections to transition equations.

\section{Abbreviations}

KDO: turbulent kinetic energy dependent only; CFD: Computational Fluid Dynamics; CAD: Computer-Aided Design.

\section{Acknowledgements}

Authors are grateful to research associate Xinghao Xiang of CARDC and professor Jiakuan Xu of NWPU for the in-depth discussion of cross-flow transition.

\section{Authors' contributions}

The contribution of the authors to the work is equivalent. All authors read and approved the final manuscript.

Funding

We gratefully thank the National Numerical Wind tunnel Project (No. NNW2019ZT3-A14) for supporting this research. This research was supported by the high performance computing (HPC) resources at Beihang University.

Availability of data and materials

All data generated or analyzed during this study are included in this published article.

\section{Declarations}

Competing interests

The authors declare that they have no competing interests.

\section{Author details}

'State Key Laboratory of Shale Oil and Gas Enrichment Mechanisms and Effective Development, Sinopec Research Institute of Petroleum Engineering, Beijing 100029, China. ${ }^{2}$ School of Energy and Power Engineering, Beihang University, Beijing 100191, China. ${ }^{3}$ Hangzhou Innovation Institute Yuhang, Beihang University, Hangzhou 311100, China. 
Received: 17 August 2021 Accepted: 25 October 2021

Published online: 14 January 2022

\section{References}

1. Aupoix B, Spalart PR (2003) Extensions of the Spalart-Allmaras turbulence model to account for wall roughness. Int $J$ Heat Fluid Flow 24(4):454-462

2. Nikuradse J (1933) Stromungsgesetze in rauhen Rohren. Tech. Rept 361, VDI-Forschungsheft

3. Dirling RB Jr (1973) A method for computing roughwall heat transfer rates on reentry nosetips. Paper presented at the 8th thermophysics conference, AIAA 1973-763. Palm Springs, CA, 16-18 July 1973

4. Grabow RM, White CO (1975) Surface roughness effects nosetip ablation characteristics. AIAA J 13(5):605-609

5. Xiang $X$, Zhang $Y$, Yuan $X$ et al (2021) $C-\gamma$-Re $e_{\theta}$ model for hypersonic three-dimensional boundary layer transition prediction. Acta Aeronaut Astronaut Sin 42(9):625711

6. Liu Z, Zhao Y, Chen S, Yan C, Cai F (2020) Predicting distributed roughness induced transition with a four-equation laminar kinetic energy transition model. Aerosp Sci Technol 99:105736

7. Wilcox DC (1988) Reassessment of the scale-determining equation for advanced turbulence models. AlAA J 26(11):1299-1310

8. Xu J, Zhang Y, Bai J (2015) One-equation turbulence model based on extended Bradshaw assumption. AlAA J 53(6):1433-1441

9. Xu J, Xu D, Zhang Y, Bai J (2019) Capturing transition with flow-structure-adaptive KDO RANS model. Aerosp Sci Technol 85:150-157

10. Chedevergne F, Aupoix B (2017) Accounting for wall roughness effects in turbulence models: a wall function approach. Paper presented at the 7th European conference for aerospace sciences (EUCASS), Milan, Italy, 3-6 July 2017

11. Schlatter P, Örlü R (2010) Assessment of direct numerical simulation data of turbulent boundary layers. J Fluid Mech 659:116-126

12. Blanchard A (1977) Analyse expérimentale et théorique de la structure de la turbulence d'une couche limite sur paroi rugueuse. Dissertation, Université de Poitiers

13. Hosni MH, Coleman HW, Taylor RP (1993) Measurement and calculation of fluid dynamic characteristics of roughwall turbulent boundary-layer flows. J Fluid Eng 115(3):383-388

14. Suga K, Craft TJ, lacovides H (2006) An analytical wall-function for turbulent flows and heat transfer over rough walls. Int J Heat and Fluid FI 27(5):852-866

15. Kreplin HP, Vollmers H, Meier HU (1985) Wall shear stress measurements on an inclined prolate spheroid in the DFVLR $3 \mathrm{~m} \times 3 \mathrm{~m}$ low speed wind tunnel, Göttingen. DFVLR-AVA, Report IB 22-84 A 33

\section{Publisher's Note}

Springer Nature remains neutral with regard to jurisdictional claims in published maps and institutional affiliations.

\section{Submit your manuscript to a SpringerOpen ${ }^{\circ}$ journal and benefit from:}

Convenient online submission

Rigorous peer review

- Open access: articles freely available online

- High visibility within the field

Retaining the copyright to your article

Submit your next manuscript at $\boldsymbol{\nabla}$ springeropen.com 